\title{
Study of variation of temperature of black holes with respect to mass in XRBs
}

\author{
Rama Nand Mehta ${ }^{1}$, Umakant Prasad ${ }^{2}$, Ashok Kumar ${ }^{3}$, Dipo Mahto ${ }^{4,}$ * \\ ${ }^{1}$ University Department of Mathematics, T. M. B. U. Bhagalpur-812007, India \\ ${ }^{2}$ Department of Physics, T.N.B. College, T. M. B. U. Bhagalpur-812007, India \\ ${ }^{3}$ Department of Physics, M.R.J.D. College, Bishnupur, Begusarai-851101, India \\ ${ }^{4}$ Department of Physics, Marwari College, T.M.B.U. Bhagalpur-812007, India
}

\section{Email address:}

rnm1k@yahoo.co.in(R. N. Mehta), umakant.prasad@gmail.com(U. Prasad),drashokbgs259@gmail.com(A. Kumar), dipomahto@hotmail.com(D. Mahto)

\section{To cite this article:}

Rama Nand Mehta, Umakant Prasad, Ashok Kumar, Dipo Mahto. Study of Variation of Temperature of Black Holes with Respect to Mass in XRBs. International Journal of Astrophysics and Space Science. Vol. 1, No. 4, 2013, pp. 61-63. doi: 10.11648/j.ijass.20130104.18

\begin{abstract}
In the present paper, we have derived an expression for the variation of temperature of the black holes with respect to mass using the temperature of black hole at which the black hole radiates to infinity all species of particles with a perfect black body spectrum and surface gravity for Schwarzschild black holes. We have also calculated their values of different test black holes existing only in X-ray binaries.
\end{abstract}

Keywords: Surface gravity, X-ray binaries, Schwarzschild black holes

\section{Introduction}

Classically black holes are perfect absorbers, but do not emit anything; their physical temperature is absolute zero (Wald,2001) and they pull all the things towards themselves like materials, messages, information, sound light etc. In 2005, Ram et. al concluded that the black hole is a Bose-Einstein ensemble of quanta of mass equal to twice the Planck mass, confined in a sphere of radius twice the black hole (Ram et al. 2005). Quantum mechanically, however, there is a possibility that one of a particle production pair in a black hole is able to tunnel the gravitational barrier and escapes the black hole's horizon. Thus, a black hole is not really black; it can radiate or evaporate particles (Triyanta et al. 2013). Stephan Hawking provided a theoretical argument for its existence in 1974, and sometimes also after the physicist Jacob Bekenstein who predicted that black holes should have a finite, non-zero temperature and entropy and also stated that it is proportional to the black hole area A (Hawking, 1988). Later on, Bardeen, Carter and Hawking performed calculations using a semi-classical approximation, putting Bekenstein conjecture on a firm basis. They established that the black hole temperature is proportional to its surface gravity (Bardeen et al.1973 \& Silva 2006). Shunichiro Kinoshita and Norihiro Tanahashi discussed the
Hawking temperature of near-equilibrium black holes using a semi-classical analysis and found that the temperature is determined by the surface gravity of the past horizon (S. Kinoshita \& N. Tanahashi, 2012). In 2013, Triyanta \& Anike N. Bowaire concluded that in the case of the Schwarzschild black hole, a higher Hawking temperature corresponds to a lower mass of a black hole (Triyanta et al. 2013).

In the present research work, an expression for the variation of temperature of the black holes with respect to mass has been derived using the temperature of black hole at which the black hole radiates to infinity all species of particles with a perfect black body spectrum and surface gravity for Schwarzschild black holes. Thereafter, we have also calculated their values of different test black holes existing only in X-ray binaries.

\section{Theoretical Discussion}

On the basis of quantum field theory, Stephan Hawking predicted that black holes should radiate energy like a black body with a temperature proportional to the surface $\operatorname{gravity}(\boldsymbol{\kappa})$ of the black hole given by 


$$
T=\frac{\kappa}{2 \pi}
$$

Thus, $\frac{\kappa}{2 \pi}$ truly is the physical temperature of a black hole, not merely a quantity playing a role mathematically analogous to the temperature in the laws of black hole mechanics (Wald 2001, Mahto et al 2012).

For the Schwarzschild case, the surface gravity $(\boldsymbol{K})$ of a black hole is given by (Transchen 2000, Mahto et al. 2011 \& 2012)

$$
\kappa=1 / 4 M
$$

Putting eq ${ }^{\text {n. }}(2)$ in eq ${ }^{\text {n. }}(1)$, temperature of a black hole becomes

$$
T=1 / 8 \pi M
$$

Using the proper value of four fundamental constants of nature - the speed of light (c), Planck's constant (h), Newton's gravitational constant $(\mathrm{G})$ and Boltzmann constant (k) on right side of the above equation, temperature of a black hole becomes

$$
\begin{gathered}
T=\frac{c h^{3}}{8 \pi k G M} \\
T=\frac{c h^{3}}{8 \pi k G}\left(M^{-1}\right)
\end{gathered}
$$

The above equation is differentiated with respect to the mass $\mathrm{M}$, we have

$$
\begin{gathered}
\frac{d T}{d M}=-\frac{c h^{3}}{8 \pi G k M^{2}} \\
\frac{d T}{d M}=-\frac{c h^{3}}{8 \pi G k}\left(\frac{1}{M^{2}}\right) \\
\frac{d T}{d M}=-K_{D}\left(\frac{1}{M^{2}}\right) \\
K_{D}=\frac{c h^{3}}{8 \pi G k} \\
K_{D}=3.767 \times 10^{-60}(\text { S.I.units }) \\
\frac{d T}{d M} \propto-\frac{1}{M^{2}}
\end{gathered}
$$

The negative sign in the equations (6) and (7) shows the decrease in mass of the black hole takes place with decrease in the temperature of black hole which occurs due to emission of the Hawking radiations from the black hole. The equation (11) shows that the variation of the temperature of the black hole is inversely proportional to the square of its mass. $K_{D}$ is the new constant involving in the equation (8) having value given by equation (10).

When particles escape, the black hole losses small amount of its energy and therefore of its mass according to mass-energy equivalence relation $\mathrm{E}=\mathrm{mc}^{2}$. The Hawking radiation process reduces the mass and energy of the black hole and is therefore also known as black holes evaporation. This also leads to the finite life time and lowering the temperature of the black holes.

\section{Data in the Support of Black Holes}

\begin{tabular}{|c|c|c|}
\hline & Variation of temp. of black $h$ & es w.r.t. mass in XRBs \\
\hline S. $\mathbf{N}$. & Mass of black holes (in $\mathbf{M}_{\square}$ ) & $\frac{d T}{d M}=-\frac{c h^{3}}{8 \pi G k M^{2}}$ ( in Kelvin $\left./ \mathbf{k g}\right)$ \\
\hline 1 & $5 \mathbf{M}_{\square}$ & $37.6667 \times 10^{-123}$ \\
\hline 2 & $6 M$ & $26.1622 \times 10^{-123}$ \\
\hline 3 & $7 \mathbf{M}_{\square}$ & $19.2251 \times 10^{-123}$ \\
\hline 4 & $\mathbf{8} \mathbf{M}$ & $14.7165 \times 10^{-123}$ \\
\hline 5 & $\mathbf{9} \mathbf{M}_{\square}$ & $11.6255 \times 10^{-123}$ \\
\hline 6 & $10 M_{\square}$ & $9.4010 \times 10^{-123}$ \\
\hline 7 & $11 \mathrm{M}_{\square}$ & $7.7831 \times 10^{-123}$ \\
\hline 8 & $12 M_{\square}$ & $6.5405 \times 10^{-123}$ \\
\hline 9 & $13 M_{\square}$ & $5.5724 \times 10^{-123}$ \\
\hline 10 & $14 M_{\square}$ & $4.8048 \times 10^{-123}$ \\
\hline 11 & $15 M_{\square}$ & $4.1859 \times 10^{-123}$ \\
\hline 12 & $16 M$ & $3.6786 \times 10^{-123}$ \\
\hline 13 & $17 M_{\square}$ & $3.2584 \times 10^{-123}$ \\
\hline 14 & $18 M_{\square}$ & $2.9064 \times 10^{-123}$ \\
\hline 15 & $19 M_{\square}$ & $2.6082 \times 10^{-123}$ \\
\hline 16 & $\mathbf{2 0 M}$ & $2.3167 \times 10^{-123}$ \\
\hline
\end{tabular}

There are two categories of black holes classified on the basis of their masses clearly very distinct from each other, with very different masses $\mathrm{M} \sim 5-20 \mathrm{M} \odot$ for stellar - mass black holes in X-ray binaries and $\mathrm{M} \sim 106-109.5 \mathrm{M} \odot$ for super massive black holes in Active Galactic Nuclei (Narayan, 2005 \& Mahto et al. 2012).

\section{Data Used in Calculation}

$\mathrm{G}=6.673 \times 10^{-11} \mathrm{~m}^{3} \mathrm{~kg}^{-1} \mathrm{~s}^{-2}, \quad \mathrm{~h}=6.623 \times 10^{-34} \mathrm{Js}$, $\mathrm{k}=1.380 \times 10^{-23} \mathrm{~J} / \mathrm{K}, \mathrm{c}=3 \times 10^{8} \mathrm{~m} / \mathrm{s}, \mathrm{M}_{\odot}=1.99 \times 10^{30} \mathrm{~kg}$.

\section{Table}




\section{Graph}

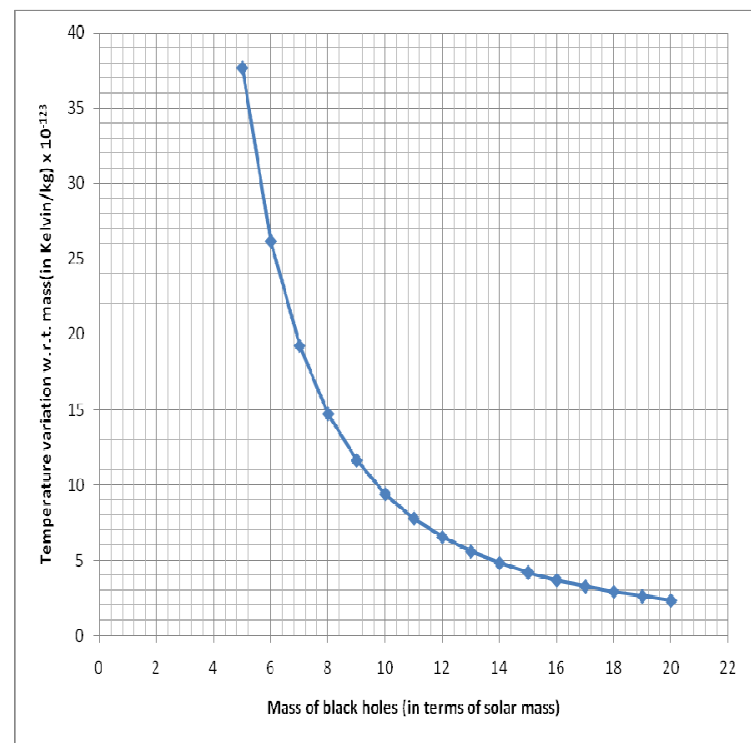

Fig 1. Graph plotted between mass of different test of black holes and variation of temp. w. r. t. mass in XRBs.

\section{Results and Discussion}

In the present paper, we have derived an expression for the variation of temperature of the black holes with respect to mass using the temperature of black hole $(T=\kappa / 2 \pi)$ at which the black hole radiates to infinity all species of particles with a perfect black body spectrum and surface gravity $(\kappa=1 / 4 M$ ) for Schwarzschild black holes and finally equation (7) is obtained. From the expression (11), it is clear that the variation of the temperature of the black hole is inversely proportional to the square of its mass and the negative sign shows the decrease in the mass of the black hole with decrease in the temperature of different test black holes. The work is further extended to calculate their values of different test black holes existing only in X-ray binaries. To know the nature of temperature variation w.r.t. mass of black holes, the graph is plotted between the mass of different test black holes and their corresponding temperature variation in XRBs (Figure 1). From the graph plotted in the figure1, it is clear that the rate of change in temperature of black holes with respect to the mass initially falls rapidly with increasing the mass of black holes from $5 M_{\odot}$ to $10 M_{\odot}$ and then gradually falls up to the mass $20 M_{\odot}$ in XRBs. The graph plotted between mass of different test of black holes and variation of temperature with respect to mass in XRBs also shows that the temperature of the black hole is lowering with increasing the mass of different test black holes. This indicates that the heavier black holes have relatively lower temperature than that of the lighter black holes which agrees with the work of Triyanta \& A. N. Bowaire, 2013 as discussed in the introduction of the same paper. This gives justification for the formula of the temperature of black hole as proposed Stephan Hawking.

\section{Conclusion}

In the light of the present work, we can draw the following conclusions:

(1) Heavier the black holes lower is the rate of change in temperature with respect to the mass of black holes.

(2) Lighter black holes radiate energy faster than heavier black holes.

(3) Our results agree with the works of Triyanta \& A. N. Bowaire and the Hawking temperature.

\section{Acknowledgement}

The authors are grateful to the referee for pointing out the errors in the original manuscript and making constructive suggestions and Prof. Gopi Kant Jha for his inspiration and motivation.

\section{References}

[1] R. M. Wald: "The thermodynamics of black holes", Living reviews in relativity, 2001.

[2] Stephen Hawking: A brief History of Time, Bantam books, 1988.

[3] Triyanta \& A. N. Bowaire: Hawking Temperature of the Reissner-Nordstrom-Vaidya Black Hole, J. Math. Fund. Sci., Vol. 45, No. 2, 2013, 114-123.

[4] Bardeen, J.M., Carter, B., Hawking, S.W.: The four laws of black hole mechanics. Commun. Math. Phys. 31(2), 161-170(1973). doi:10.1007/BFO1645742.

[5] P. R. Silva: "A Note on Black Hole Temperature and Entropy". General Relativity and Quantum Cosmology, arXiv.org/pdf/gr-qc/0605051, May, 2006.

[6] B. Ram, A. Ram and N. Ram: "The quantum black hole"arXiv:gr-qc/0504030v1.April, 2005.

[7] D. Mahto, V. Prakash, B. K. Singh, K.M. Singh: Change in entropy of Non-spinning black holes w.r.t. the radius of event horizon in XRBs., Astrophys Space Sci. DOI 10.1007/s10509-012-1219-y (2012).

[8] J. Transchen,: "An introduction to black hole evaporation" (2000), arXiv: gr- qc/0010055V1.

[9] R. Narayan: Black Holes In Astrophysics, New Journal Physics, Vol. 7,No. 1, 2005, pp1-31, arXiv: gr-qc/050 6078.

[10] D. Mahto, K. Kumari, R. K. Sah \& K. M. Singh: Study of non-spinning black holes with reference to the change in Energy and Entropy, Astrophys Space Sci. DOI: 10.1007/s10509-011-0883-7 (2011).

[11] S. Kinoshita and N. Tanahashi: "Hawking temperature for near-equilibrium black holes." arXiv: 1111.2684v2 [hep-th], 27 Jan, 2012. 